\section{Mitral valve surgery with epicardial cryoablation efficaciously treats AF}

Atrial fibrillation (AF) often persists after mitral valve surgery (MVS); surgeons are trying to improve success rates by modifying the surgical procedure. Cox-Maze III surgery performed at the same time as MVS might be more effective than MVS alone but involves complex incisions. Although epicardial cryoablative surgery seems promising, no randomized trials have yet compared MVS with MVS plus cryoablation.

Blomström-Lundqvist et al. report a trial involving 69 patients with permanent AF, 35 of whom were randomly assigned to receive MVS alone, and 34 of whom underwent MVS with epicardial left atrial cryoablation. After 6 months, 22 (73.3\%) patients in the MVScryoablation group had regained sinus rhythm compared with 16 (45.7\%) patients in the MVSonly group. At 12 months' follow-up, all of the 22 patients in the MVS-cryoablation group had maintained sinus rhythm compared with 15 in the MVS-only group. Coronary artery disease was identified as a risk factor for failure of cryoablation to prevent AF after MVS.

This prospective, randomized, multicenter, controlled trial -the first of its kind in patients with AF-shows that MVS performed concomitantly with epicardial left atrial cryoablation is more effective than MVS alone. This modified technique restores and maintains sinus rhythm in patients with permanent AF, but the authors stress that larger studies are now needed to confirm the method's long-term efficacy and safety.

Original article Blomström-Lundqvist C et al. (2007) A randomized double-blind study of epicardial left atrial cryoablation for permanent atrial fibrillation in patients undergoing mitral valve surgery: the SWEDish Multicentre Atrial Fibrillation study (SWEDMAF). Eur Heart $J$ 28: 2902-2908

\section{Unfit older adults have higher mortality risk than their fitter peers, regardless of obesity}

The burgeoning elderly population and the increasing prevalence of obesity and physical inactivity in the US are putting a substantial burden on health-care services. Obese, inactive, middle-aged adults are at high risk of early death, but few studies have investigated the link between obesity, physical activity levels, and survival in older adults. Sui et al. looked at the relationship between these elements and at the impact of fitness by studying 2,603 adults aged 60 years or older who were taking part in the Aerobics Center Longitudinal Study.

During the 12-year follow-up, 450 of the participants died. Analysis of data including BMI, waist circumference and percentage body fat revealed that mortality correlated with $\mathrm{BMI}$ and waist circumference, but not with percentage body fat. The death rate per 1,000 personyears in individuals with a $\mathrm{BMI}$ in the range $18.5-24.9 \mathrm{~kg} / \mathrm{m}^{2}$ was 13.9 , compared with 31.8 in the group with a BMl over $35 \mathrm{~kg} / \mathrm{m}^{2}$. Waist circumferences of $\geq 88 \mathrm{~cm}$ for women and $\geq 102 \mathrm{~cm}$ for men were risk factors for mortality after adjustment for smoking, baseline health status, and BMI. Poor fitness was strongly associated with mortality even after adjustment for potential confounding factors, including various measurements of adiposity.

The authors conclude that older adults who retain fitness are likely to live longer than those who are unfit, irrespective of the weight and fat distribution of the individual. They suggest that mortality in this age-group could be reduced by the promotion of regular physical activity.

Original article Sui X et al. (2007) Cardiorespiratory fitness and adiposity as mortality predictors in older adults. JAMA 298: $2507-2516$

\section{Hypertension could increase risk of developing nonamnestic mild cognitive impairment}

Evidence indicates that mild cognitive impairment $(\mathrm{MCl})$ might herald the development of Alzheimer's disease in certain patients. Annual conversion rates to Alzheimer's disease of between $10 \%$ and $12 \%$ have been observed in patients with $\mathrm{MCl}$. Vascular dementia, $\mathrm{MCl}$ and Alzheimer's disease are thought to be related to hypertension, a potentially modifiable risk factor, although the exact associations are unclear. To investigate the possible link between hypertension and $\mathrm{MCl}$, Reitz et al. followed up a community-based cohort of 918 people for a total of 4,337 person-years.

In total, 334 cases of incident $\mathrm{MCl}$ were identified, 160 of which were classified as amnestic and 174 as nonamnestic. After adjustment 